\title{
Esperamicin A1
}

National Cancer Institute

\section{Source}

National Cancer Institute. Esperamicin A1. NCI Thesaurus. Code C1301.

An enediyne antineoplastic antibiotic hybrid containing an anthranilate moiety.

Esperamicin A1 is isolated from the bacterium Actinomadura verrucosospora. The anthranilate component of esperamicin A1 intercalates DNA and the benzene diradical intermediate of the enediyne core binds to the minor groove of DNA, resulting in singleand double-stranded breaks in DNA and apoptosis. (NCI04) 